\title{
BMJ Open Determinants of postnatal depression in Sudanese women at 3 months postpartum: a cross-sectional study
}

\author{
Dina Sami Khalifa, ${ }^{1,2}$ Kari Glavin, ${ }^{3}$ Espen Bjertness, ${ }^{1}$ Lars Lien ${ }^{4,5}$
}

\begin{abstract}
To cite: Khalifa DS, Glavin K, Bjertness $\mathrm{E}$, et al. Determinants of postnatal depression in Sudanese women at 3 months postpartum: a cross-sectional study. BMJ Open 2016;6: e009443. doi:10.1136/ bmjopen-2015-009443
\end{abstract}

- Prepublication history for this paper is available online. To view these files please visit the journal online (http://dx.doi.org/10.1136/ bmjopen-2015-009443).

Received 21 July 2015 Revised 11 December 2015 Accepted 19 January 2016

\section{CrossMark}

\footnotetext{
${ }^{1}$ Department of Community Medicine, Institute of Health and Society, Faculty of Medicine, University of Oslo, Oslo, Norway

${ }^{2}$ Faculty of Health Sciences, Ahfad University for Women, Khartoum, Sudan

${ }^{3}$ Diakonova University

College, Oslo, Norway

${ }^{4}$ National Advisory Board on Dual Diagnosis, Innlandet Hospital Trust, Hamar, Norway

${ }^{5}$ Department of Public Health, Hedmark University College, Elverum, Norway
}

Correspondence to Dr Dina Sami Khalifa; dinasami5071@hotmail.com

\section{ABSTRACT}

Objectives: Maternal mental health is a neglected issue in Sudanese healthcare. The aim of this study was to explore the factors associated with postnatal depression (PND) at 3 months postpartum in a sample of Sudanese women in Khartoum state.

Setting: Recruitment was from two major public antenatal care (ANC) clinics in two maternity teaching hospitals in Khartoum state. The study participants were recruited during their pregnancy and were followed up and screened for PND at 3 months postpartum using the Edinburgh Postnatal Depression Scale (EPDS).

Participants: A sample of 300 pregnant Sudanese women in their second or third trimester was included in the study. The inclusion criteria were Sudanese nationality, pregnancy in the second or third trimester and satisfactory contact information.

Outcome measures: PND was assessed using the EPDS at a cut-off score of $\geq 12$. Maternal and sociodemographic factors of interest were illustrated in a directed acyclic graph (DAG) to identify which variables to adjust for in multivariate analyses and to show their type of effect on PND. A forward logistic regression model was built to assess the factors that are independently associated with PND.

Results: History of violence increased the odds of PND sevenfold, OR=7.4 (95\% Cl 1.9 to 27.6). Older age of mothers decreased the odds of PND by almost $20 \%, 0 \mathrm{R}=0.82(95 \% \mathrm{Cl} 0.73$ to 0.92$)$. Exclusive breast feeding and regular prenatal vitamins during pregnancy are associated with an $80 \%$ decrease in odds of PND, $\mathrm{OR}=0.2(95 \% \mathrm{Cl} 0.06$ to 0.70$)$ and $0.17(95 \% \mathrm{Cl} 0.06$ to 0.5$)$, respectively.

Conclusions: Factors associated with PND in this study are comparable to factors from other developing countries, although findings should be judged with caution owing to the high number of women who refused recruitment into the study.

\section{INTRODUCTION}

The WHO declared that one in four individuals will develop a mental or behavioural disorder during their lifetime, and that 20-40\% of women in developing countries experience depression during pregnancy or after

\section{Strengths and limitations of this study}

- This was the first study in Sudan addressing maternal mental health by means of a screening tool (EPDS) that we validated in a Sudanese setting.

- Recruitment during pregnancy maximised follow-up and screening at 3 months postpartum.

- Our study assessed postnatal depression beyond the puerperal period (the period during which Sudanese women receive the most support after birth).

- We provided an interpretation on the type of effect each variable had on depression status using directed acyclic graphs (DAGs).

- A high number of women refused inclusion into the study.

childbirth. ${ }^{1}$ The estimated average prevalence of perinatal mental disorders in the Eastern Mediterranean region (EMR) is $15-36 \% .^{2}$ Women suffering from mental illness during pregnancy, specifically depression or psychosis, will have limited caregiving capabilities, thus leading to child neglect and future developmental and behavioural problems. ${ }^{3}$

Prevalence figures of postnatal depression (PND) are variable across different settings and cultures owing to variations in socioeconomic and gender-based determinants. ${ }^{4}$ Research in low-income countries presented opposite points of view regarding this variation. Qualitative research illustrated that pregnant and postpartum women in low-income countries are protected from perinatal mental illness owing to the social construct and the 'traditional ritualised support' they receive during and after pregnancy. They suggest that this type of support is missing in higher-income countries. ${ }^{5}$ In contrast, quantitative research in low-income countries continues to measure a similar or higher prevalence of PND compared with high-income countries. They suggest this is because of the environment in which women 
are reproducing, an environment of 'depression-related risk factors'. ${ }^{7}$ They are living in circumstances of poverty, low educational level, early marriage, limited decision-making authority concerning reproductive autonomy, poor nutritional status and other genderbased stereotypical roles and responsibilities. ${ }^{7}$ In a country such as Sudan, the amount of social support pregnant women receive is substantial; however, women are also living in a male-dominated society and in the middle of conflict situations, migration and deteriorating economic status, thereby making women highly susceptible to mental illness. ${ }^{8}$ Hence, pregnancy-related mental health problems are expected to be higher among women in such settings.

Existing knowledge shows variations in the prevalence of PND among low-income countries despite comparably associated factors. In Ghana, the 1-month prevalence of PND is $3.8 \%$, with antenatal depression and adverse birth outcomes as associated risk factors. ${ }^{9}$ In the United Arab Emirates (UAE), $16.8 \%$ exhibited symptoms of PND at 2 months postpartum when screened with the Edinburgh Postnatal Depression Scale (EPDS). Antenatal depression, parity, religion and the use of formula for feeding are the significant risk factors. ${ }^{10}$ In Lebanon, the prevalence of PND was $12.8 \%$ at $30-40$ days after delivery, with a past history of depression being a significant risk factor. ${ }^{11}$ In Uganda, the 3-month prevalence was as high as $43 \%$, while associated risk factors were bad marital relations, mother's parity and infant characteristics. ${ }^{12}$ In Upper Egypt, mothers screened for PND up to 1 year after birth had a high prevalence of $49 \% .{ }^{13}$ A low household income, child sleeping hours, complications after delivery and a lack of support from the husband after delivery were found to be statistically associated with PND. ${ }^{13}$

Caring for mothers with mental illness is neglected in healthcare systems in low-income countries, with Sudan being a prime example. This is because of the lack of understanding of the nature of maternal depression among women and among primary healthcare providers. In addition, the stigma associated with the condition prevents women from accessing mental healthcare. The neglect of maternal mental health issues could be an important reason that Sudan failed to reach its Millennium Development Goals (MDGs) of reducing maternal mortality ratio and the under-five mortality rate. ${ }^{14}$ Accordingly, maternal mental health should be a research focus in Sudan. The prevalence of PND at 3 months after birth was reported from a previous analysis from this study by Khalifa $e t a l^{15}$ as $9.2 \%$. Therefore, the aim of this analysis is to investigate factors associated with PND in the same cohort of Sudanese women.

\section{METHODOLOGY}

Study design

This was a cross-sectional study of 300 participants recruited during pregnancy with demographic data and contact information collected at recruitment. At
3 months after delivery, we screened 238 participants for PND with the EPDS at a cut-off score of $\geq 12$. We also collected data on the proposed factors of associations (eg, obstetrical and medical complications during pregnancy or birth, place of delivery, regular uptake of vitamins during pregnancy, sex of the newborn, puerperal complications and circumstances after birth). The sample size was calculated using the prevalence of PND in Nigeria, a neighbouring African country with a similar social context to Sudan. ${ }^{16}$ The prevalence of PND in Nigeria is comparable with the pooled prevalence of postpartum common mental disorders reported in 2012 by the WHO's systematic review of perinatal mental disorders from developing and underdeveloped countries. ${ }^{6}$

Women presenting at two antenatal clinics in two major public tertiary hospitals were invited to participate in the study, and the hospitals chosen were Omdurman Maternity Teaching Hospital (90\% of total sample) and Ibrahim Malik Teaching Hospital (10\% of the sample). Khartoum state has the highest level of utilisation of antenatal care (ANC) services in Sudan and the highest level of institutionally based deliveries as well. ${ }^{17}$ ANC attendance in Khartoum state is $88 \%,{ }^{17}$ which indicates the proportion of women who attend 'at least one' ANC visit with a skilled provider during a pregnancy. Women from all localities of Khartoum state can access ANC services in Omdurman Maternity Hospital because access does not depend on the location of residence. ${ }^{18}$ The inclusion criteria were women of Sudanese nationality residing in Khartoum state, in the second or third trimester, of any parity and with full contact information (at least two working telephone numbers). Illiteracy was not an exclusion criterion as data collection was via interviews.

\section{Procedure}

Recruitment was intermittent during the period from April 2013 to April 2014. Hospital records showed that almost 5000 women attended the clinics during that year. We approached candidates after the completion of their physical examination. The examining physician introduced the principal investigator to each candidate, and we approached and assessed 700 pregnant women for eligibility (figure 1). Two hundred women were excluded owing to insufficient contact information (unavailability of mobile or home phone numbers), a non-Sudanese nationality and being in their first trimester. Among the 500 women who satisfied inclusion criteria, 200 refused to participate in the study. The final sample for follow-up was $300(60 \%)$, that is, the first interview (T0). A total of 238 women completed the follow-up at 3 months postpartum, that is, the second interview (T1). Last, no information was available for us on the 200 women that refused participation into the study.

First interview (TO, $\mathrm{n}=300)$

Information was collected at recruitment on sociodemographic data, as full contact information was obtained at 


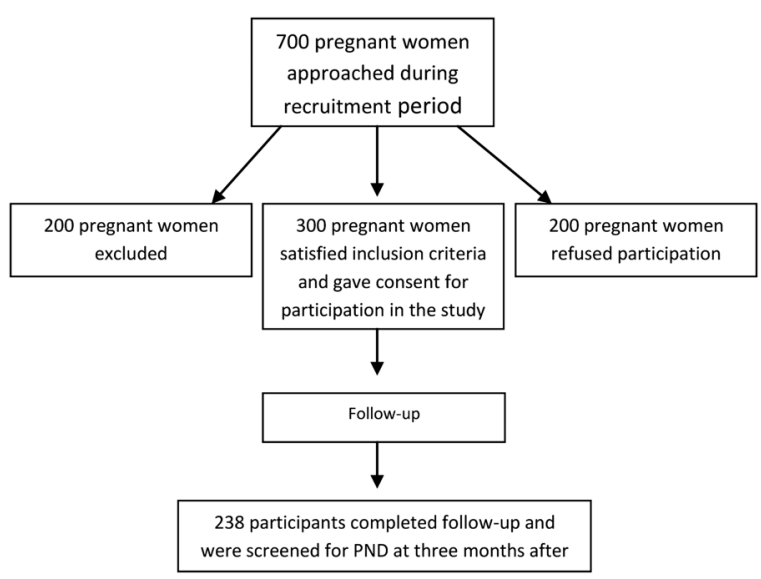

Figure 1 Flow chart of the number of participants in the study.

that time to secure follow-up and screening for PND after delivery.

\section{Second interview $(\mathrm{T} 1, \mathrm{n}=238)$}

At 3 months, 238 participants were screened for PND with the EPDS. ${ }^{15}$ Interviews were done either face-to-face or through phone interviews. Phone interviews were conducted to minimise the loss of follow-up only when women were away from Khartoum state or refused home visits. As reported from a previous analysis in the same study, ${ }^{15}$ the follow-up rate at 3 months postpartum was $79 \%$. Moreover, the loss to follow-up was due to personal refusal $(4.7 \%)$, the husband's refusal $(4.7 \%)$ and contact failure $(11.3 \%)$. Participants who lost to follow-up were not significantly different from women who completed the follow-up in age (the mean age was 27 and 28 years, respectively), in parity (the median parity was 1.9 children and 1.8 children, respectively) or in educational level (Pearson $\chi^{2} \mathrm{p}$ value $\left.=0.705\right)$.

\section{Measures}

The Edinburgh Postnatal Depression Scale

The EPDS is a reliable and validated screening tool for PND developed for use at the primary healthcare level ${ }^{19}$ and has been translated and validated into 57 languages, including Arabic. ${ }^{20}$ It is a screening test consisting of 10 inventory questions that investigate feelings occurring within the previous 7 days with each question having 4 possible answers rated from 0 to 3 . A woman is considered 'test positive' for PND if she scores 12 or more out of 30 as set by Cox et $a l^{19}$ The tool was originally designed to be self-administered, but studies have shown that screening through directed interviews is an equivalent screening technique. ${ }^{21}$ Ghubash et al, the first authors to translate the EPDS into the Arabic language, stated that EPDS had a Cronbach's coefficient of 0.84 . In the current study, the Cronbach's coefficient of the EPDS is 0.83 .

\section{Determinants of interest}

In addition to sociodemographic information, data on certain variables of interest were collected. Data were collected on the history of any psychological condition, history of violence, place of stay after birth, supportive person after birth, newborn gender and characteristics, complication during pregnancy and birth, planning of current pregnancy, regular uptake of prenatal vitamins, breastfeeding practices, circumstances during and after pregnancy and satisfaction with current quality of life.

\section{Statistical analysis}

The analysis in this article was done after validation of the EPDS screening tool on the same sample. We constructed an initial directed acyclic graph (DAG) for a number of variables of interest (see figure 2). The aim of the analysis was to investigate multiple independent associations with PND using logistic regression. Crude measures of association between each variable and PND were first analysed by $\chi^{2} / \mathrm{t}$ tests and Mantel-Haenszel (crude) ORs. A multivariable regression model was then built using a forward regression selection approach. The variable with the smallest overall $p$ value from the crude analysis was selected first and incorporated into the model. Next, each variable was included in the model in turn and a likelihood ratio test (LRT) was performed. The process was repeated until only variables with p $>0.05$ remained. The model was based on complete observations on all variables of interest, and interaction between the final variables in the model was also tested. A DAG was drawn with the model variables to interpret the type of effect each variable has on PND.

\section{RESULTS}

Out of 238 women included in the present study, we have reported in a previous validation study that 22 women screened positive for PND with the EPDS at a cut-off point of $\geq 12$, thus resulting in a prevalence of $9.2 \%{ }^{15}$ About $12 \%$ of study participants held an occupation at the time of recruitment. The majority of recruited women $(72 \%)$ were unemployed with no history of employment, $10 \%$ had an occupation before marriage and $6 \%$ were students. About $2 \%$ had no formal education (illiterate), whereas $39 \%$ had university and/or postgraduate education. The mean age of participants was 28 years, with $41 \%$ between the ages of 15 and 25. Current pregnancy was unplanned in $35 \%$ of women, $6 \%$ had home deliveries, almost $40 \%$ delivered by caesarean section and $8 \%$ were in a polygamous marriage. Table 1 illustrates descriptive statistics of women screened for PND at 3 months postpartum (test positive vs test negative women) at a prevalence of $9.2 \%$.

\section{Significant factors by univariable analysis}

A univariable analysis revealed that 10 variables are associated with PND using $\chi^{2}$ tests (for categorical variables) and $\mathrm{t}$ tests (for continuous variables) at a significance 
Figure 2 A DAG of the factors of interest in the analysis. DAG, directed acyclic graph.

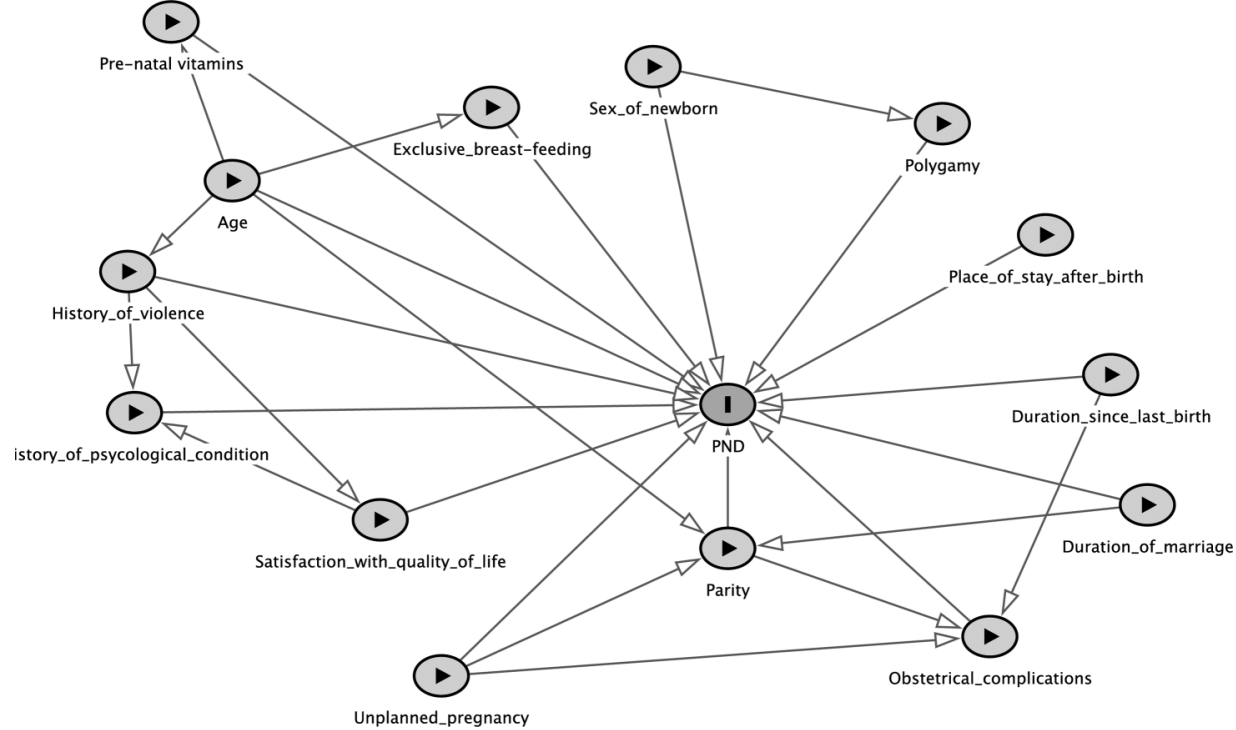

level of 0.05 (table 1). The analysis showed no significant relationships between PND and any obstetrical complications and most sociodemographic variables. Also, newborn sex and characteristics were not associated with PND. Variables increasing the odds of depression were younger maternal age, shorter duration of marriage, previous history of a psychological condition, history of violence/stress and dissatisfaction with living conditions. Factors reducing the odds of PND were exclusive breast feeding, regular prenatal vitamins during pregnancy (regular uptake for at least two trimesters), higher parity, longer duration since last birth and place of stay during puerperium (family home vs own home). Table 2 illustrates the crude ORs of variables significantly associated with PND.

The forward regression selection model building approach resulted in four factors independently associated with PND: age in years, history of violence, exclusive breast feeding and regular prenatal vitamins (table 3). Models with and without interaction terms were compared. Interaction was not significant at the $5 \%$ level, and proportional odds were assumed in the final model. Figure 3 illustrates the DAG of the variables in the final regression model.

\section{Effects of factors independently associated with PND}

Violence is associated with PND irrespective of the age of the woman. Women who suffered domestic violence before or during pregnancy have a sevenfold increase in the odds of depression at 3 months postpartum, whereas women who took prenatal vitamins for at least two trimesters during pregnancy have more than $80 \%$ less odds of PND irrespective of their age. Exclusive breast feeding for the first 3 months drops the odds of PND by $80 \%$. A younger age for women is associated with PND, and an increase in age of a woman decreases her odds by almost $20 \%$ per year. The effect of age on the odds of
PND is a direct effect that does not pass through any of the mediators in the DAG. The effect of a 'history of violence', 'exclusive breast feeding' and 'prenatal vitamins' is the same across all pathways; hence, their effect on PND is a total effect.

\section{DISCUSSION AND RECOMIMENDATIONS}

According to the literature, the peak time for PND is 6-8 weeks, but we hypothesised that PND among owing to the care and support they receive during puerperium from their families and communities. Postpartum women across different cultures and socioeconomic backgrounds are susceptible to PND which suggests a diversity in determinants. ${ }^{4}$ More than 70 predictors of PND are reported in developing countries. ${ }^{4}$ The strongest determinant for PND in this study, a 'history of violence', has been highlighted in individual studies and in systematic reviews as one of the strongest predictors of PND. ${ }^{22-25}$ These studies have stated that there is "consistency in the reporting of domestic violence among depressed mothers across cross-sectional studies'. ${ }^{22}$ There are no published estimates on genderbased or domestic violence in Sudan. Several studies have documented younger maternal age and first pregnancy as determinants of PND, particularly among adolescent mothers. ${ }^{26} 27$ The percentage of girls between the ages of 15 and 19 who had at least one child in Khartoum state in 2010 is $10 \%,{ }^{17}$ with the figure reaching $30 \%$ in Darfur states. ${ }^{17}$ Consequently, younger mothers and primigravidas should receive support and quality ANC to detect those at risk of PND.

Participants had lesser odds of PND when they exclusively breast fed for 3 months after birth. Studies have highlighted that breastfeeding mothers continue to score lower in depression screening tests. ${ }^{28}{ }^{29}$ Attitudes Sudanese women is more likely to peak after that period 
Table 1 Characteristics of the women screened for postnatal depression ${ }^{*}$ at 3 months postpartum $(n=238)$

\begin{tabular}{|c|c|c|c|c|}
\hline Variable & $\mathbf{N}$ & Test positive (EDPS $\geq 12$ ) & Test negative (EPDS $\leq 12$ ) & p Value \\
\hline EPDS (mean=4.4, SD=4.6) & 238 & $22($ mean=15.4, SD=3.0) & 216 (mean=3.3 SD=2.9) & $<0.001$ \\
\hline \multicolumn{5}{|l|}{ Age group $($ mean $=28, S D=5.5)$} \\
\hline Less than 19 & 8 & $2(9.1 \%)$ & $6(2.8 \%)$ & \multirow[t]{3}{*}{0.00} \\
\hline $20-29$ & 136 & $18(81.8 \%)$ & $118(54.6 \%)$ & \\
\hline More than 30 & 94 & $2(9.1 \%)$ & $92(42.6 \%)$ & \\
\hline \multicolumn{5}{|l|}{ Educational level } \\
\hline University and above & 93 & 9 (40.9\%) & $84(38.9 \%)$ & \multirow[t]{4}{*}{0.81} \\
\hline Secondary & 66 & $5(22.7 \%)$ & $61(28.2 \%)$ & \\
\hline Primary & 74 & $8(36.4 \%)$ & $66(30.6 \%)$ & \\
\hline No education & 5 & $0(0 \%)$ & $5(2.3 \%)$ & \\
\hline \multicolumn{5}{|l|}{ Occupational status } \\
\hline Never employed & 167 & $12(54.5 \%)$ & $155(71.8 \%)$ & \multirow[t]{4}{*}{0.05} \\
\hline Currently employed & 34 & $2(9.1 \%)$ & $32(14.8 \%)$ & \\
\hline Previously employed & 25 & $4(18.2 \%)$ & $21(9.7 \%)$ & \\
\hline Current student & 12 & $4(18.2 \%)$ & $8(3.7 \%)$ & \\
\hline \multicolumn{5}{|l|}{ Parity } \\
\hline Primigravida & 59 & $10(45.5 \%)$ & $49(22.7 \%)$ & \multirow[t]{3}{*}{0.03} \\
\hline Multigravida (1-4) & 158 & $12(54.5 \%)$ & $146(67.6 \%)$ & \\
\hline Grand multipra (>4) & 21 & $0(0.0 \%)$ & $21(9.7 \%)$ & \\
\hline \multicolumn{5}{|l|}{ Polygamy } \\
\hline Yes & 19 & $4(18.2 \%)$ & $15(6.9 \%)$ & \multirow[t]{2}{*}{0.10} \\
\hline No & 219 & $18(81.8 \%)$ & $201(93.1 \%)$ & \\
\hline \multicolumn{5}{|c|}{ Complications during or after pregnancy } \\
\hline Yes & 87 & $11(50.0 \%)$ & $76(35.2 \%)$ & \multirow[t]{2}{*}{0.17} \\
\hline No & 151 & $11(50.0 \%)$ & $140(64.8 \%)$ & \\
\hline \multicolumn{5}{|l|}{ Presence of chronic illness } \\
\hline Yes & 42 & $4(18.2 \%)$ & $38(17.6 \%)$ & \multirow[t]{2}{*}{0.94} \\
\hline No & 196 & $18(81.8 \%)$ & $178(82.2 \%)$ & \\
\hline \multicolumn{5}{|l|}{ Place of delivery } \\
\hline Health facility & 220 & $22(100 \%)$ & $198(91.7 \%)$ & \multirow[t]{2}{*}{0.06} \\
\hline Home & 18 & $0(0.0 \%)$ & $18(8.3 \%)$ & \\
\hline \multicolumn{5}{|l|}{ Sex of newbornt } \\
\hline Male(s) & 136 & $15(68.2 \%)$ & $121(57.1 \%)$ & \multirow[t]{2}{*}{0.31} \\
\hline Female(s) & 98 & 7 (31.8\%) & $91(42.9 \%)$ & \\
\hline \multicolumn{5}{|l|}{ Type of delivery } \\
\hline Vaginal delivery & 148 & $13(59.1 \%)$ & $135(62.5 \%)$ & \multirow[t]{3}{*}{0.95} \\
\hline $\mathrm{EmC/S}$ & 29 & $3(13.6 \%)$ & $26(12.0 \%)$ & \\
\hline Elective $\mathrm{C} / \mathrm{S}$ & 61 & $6(27.3 \%)$ & $55(25.5 \%)$ & \\
\hline
\end{tabular}

towards breast feeding in Sudanese culture are quite favourable, and it is highly prevalent in Sudan that mothers exclusively breast feed for up to 4 months $(70 \%) .^{30}$ Mothers who do not introduce formula feeding are highly praised in their communities, whereas mothers who do are criticised. The criticism of new mothers on their caring practices could heighten feelings of anxiety or depression. Another factor associated with lower odds of PND in this study was being on regular prenatal vitamins during pregnancy (for at least two trimesters in the form of folic acid and iron supplements). Women attending routine ANC in Sudan are prescribed 'Fefol', an iron and folic acid containing vitamin, as a prophylaxis for iron and folic acid deficiency anaemia during pregnancy irrespective of their blood haemoglobin level. A lower haemoglobin level is documented as a physiological factor associated with PND in a number of studies, ${ }^{31-36}$ and it is proposed that the role of iron in thyroid hormone metabolism and in the function of inflammatory cytokines might be the underlying cause of depression. ${ }^{37} 38$ In this study, haemoglobin levels were not measured. Moreover, the prevalence of iron deficiency anaemia among pregnant Sudanese women in Khartoum state is more than $30 \%,{ }^{39}$ thereby indicating the possibility that a significant proportion of the women in the study could be anaemic, and that the routine supplementation with Fefol vitamins during pregnancy reduces the odds of PND. 
Table 2 Univariable analysis of 238 screened women (22 women test positive, 216 women test negative)

\begin{tabular}{|c|c|c|c|c|}
\hline \multirow[b]{2}{*}{ Variable } & \multicolumn{2}{|c|}{ Screening status } & \multirow[b]{2}{*}{ Crude OR (95\% Cl) } & \multirow[b]{2}{*}{ p Value * } \\
\hline & Test positive & Test negative & & \\
\hline Age (years) (mean, SD)† & $24.1(3.7)$ & $28.7(5.6)$ & 0.84 (0.76 to 0.09$)$ & $<0.001$ \\
\hline Duration of marriage (years) (mean, SD)† & $2.7(3.6)$ & $6.7(5.7)$ & 0.47 (0.31 to 0.73$)$ & $<0.001$ \\
\hline \multicolumn{5}{|l|}{ Regular prenatal vitamins } \\
\hline Yes & 8 & 163 & $0.19(0.07$ to 0.48$)$ & $<0.001$ \\
\hline No & 14 & 53 & & \\
\hline \multicolumn{5}{|l|}{ History of violence (domestic/marital) } \\
\hline Yes & 6 & 15 & $5.02(1.71$ to 14.72$)$ & 0.00 \\
\hline No & 16 & 201 & & \\
\hline \multicolumn{5}{|l|}{ History of a psychological condition } \\
\hline Yes & 2 & 14 & 4.24 (1.36 to 13.20$)$ & 0.01 \\
\hline No & 17 & 202 & & \\
\hline Duration since last birth (years) (mean, SD) $\dagger$ & $2.26(1.9)$ & $3.34(2.0)$ & $0.18(0.04$ to 0.76$)$ & 0.02 \\
\hline Parity (median, IQR) $\dagger$ & $1(2)$ & $2(3)$ & 0.53 (0.30 to 0.93$)$ & 0.03 \\
\hline \multicolumn{5}{|l|}{ Exclusive breast feeding } \\
\hline Yes & 15 & 183 & $0.34(0.12$ to 0.96$)$ & 0.04 \\
\hline No (mixed/formula) & 6 & 25 & & \\
\hline \multicolumn{5}{|l|}{ Place of stay after delivery } \\
\hline Family home & 16 & 108 & $0.37(0.14$ to 0.99$)$ & 0.05 \\
\hline Own home (ref) & 6 & 108 & & \\
\hline \multicolumn{5}{|l|}{ Satisfaction with quality of life } \\
\hline Dissatisfied & 3 & 10 & $4.16(1.03$ to 16.85$)$ & 0.05 \\
\hline Neither & 5 & 12 & 5.77 (1.78 to 18.71$)$ & 0.00 \\
\hline Satisfied (ref) & 14 & 194 & & \\
\hline
\end{tabular}

Some important variables hypothesised to be associated with PND among Sudanese women before analysis were not significant after analysis (see figure 2), including 'male gender preference' and 'unplanned pregnancy'. Women giving birth to female newborns are criticised and blamed by their husbands and family members in various cultures in China, Japan, India and Turkey. ${ }^{40-44}$ Unplanned pregnancy was reported as a factor associated with PND in Indonesia and Pakistan. ${ }^{45}$ Although the uptake of family planning in Sudan is low $(10 \%),{ }^{17}$ 'unplanned pregnancy' remains a factor not associated with PND. It could be construed that Sudanese culture encourages procreation and the creation of larger families.

Socioeconomic deprivation variables such as a low educational level and unemployment were not associated with PND in our sample. This coincides with existing evidence which states socioeconomic variables play a small role in developing PND. ${ }^{47}$ Furthermore, similar to studies from other populations, obstetrical and newborn complications were not associated with PND at any level of this analysis. ${ }^{47}$ The absence of important determinants in a population is an important finding in itself, as it assists in tailoring interventions for maternal mental health.

Table 3 Crude and adjusted effects of the variables in the logistic regression model $(n=238)^{*}$

\begin{tabular}{|c|c|c|c|c|c|}
\hline Variable & Unadjusted effect (OR) & $95 \% \mathrm{Cl}$ & Adjusted effect (OR) & $95 \% \mathrm{Cl}$ & Type of effect $†$ \\
\hline Age (years) & 0.84 & (0.76 to 0.09$)$ & $0.82 \ddagger$ & (0.73 to 0.92$)$ & Direct \\
\hline History of violence & 5.02 & (1.71 to 14.72$)$ & $7.40 \S$ & (1.90 to 27.60$)$ & Total \\
\hline $\begin{array}{l}\text { Exclusive breast } \\
\text { feeding }\end{array}$ & 0.34 & $(0.12$ to 0.96$)$ & $0.20 \S$ & $(0.06$ to 0.70$)$ & Total \\
\hline $\begin{array}{l}\text { Regular prenatal } \\
\text { vitamins }\end{array}$ & 0.19 & (0.07 to 0.48$)$ & $0.17 \S$ & (0.06 to 0.50$)$ & Total \\
\hline \multicolumn{6}{|c|}{$\begin{array}{l}\text { *Interactions between the variables in the model were tested and found insignificant. } \\
\text { †According to the DAG. } \\
\text { †Adjusted for history of violence, exclusive breast feeding and regular prenatal vitamins to give a direct effect of age on PND according to the } \\
\text { DAG. } \\
\text { §Adjusted for age in years according to the DAG. } \\
\text { DAG, directed acyclic graph; PND, postnatal depression. }\end{array}$} \\
\hline
\end{tabular}




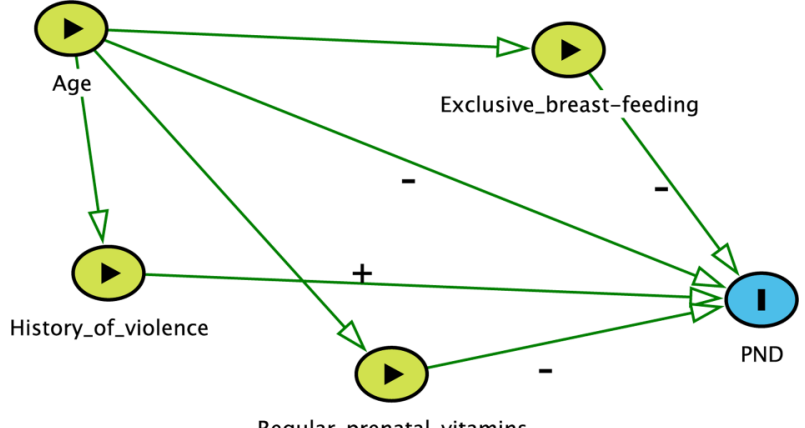

Regular_prenatal_vitamins

Figure 3 A DAG of the factors associated with PND from the logistic regression model. DAG, directed acyclic graph; PND, postnatal depression.

Sudan did not reach its target indicators in child and maternal health, which could be because of issues not addressed by the Sudanese health system, such as maternal mental health. Efforts to develop evidence-based policies to address maternal mental health in Sudan are highly needed, since there is global evidence of the long-term economic costs to neglecting maternal mental health. Nonetheless, there is evidence on the feasibility and acceptability of screening for PND in low-resource settings, especially with higher cut-off points that result in higher specificity and lower false-positive rates (A Bauer, M Parsonage, M Knapp, et al. The costs of perinatal mental health problems. Unpublished 2014) ${ }^{48}$ At low-resource settings, such as in Sudan, screening at higher cut-off points decreases the number of women requiring diagnosis by clinical examination. Clear guidelines for detection, referral and treatment of prenatal and postnatal women are needed. The early identification of 'at-risk mothers', that is, young mothers, primigravidas and women in abusive relationships, is a priority so that timely tailored counselling and treatment can be initiated. Awareness raising among Sudanese women on the mental stages that females pass through during their reproductive lives is crucial, along with awareness raising on the benefits and safety of certain medical treatments for depression during breast feeding. ${ }^{49}$ Community-based health promotion programmes could accomplish this, and we also need capacity building for healthcare personnel, particularly for those working at the community level, to remove organisational barriers to maternal mental health.

\section{Strengths and limitations}

This was the first study in Sudan addressing PND. However, measurement error of some variables is expected. Women under-report their subjection to violence, which may have underestimated the association between PND and domestic violence. Additionally, the 'history of a psychological condition' could have been under-reported. The protective effect of taking prenatal vitamins in this study is an important finding that needs further research. Owing to the acceptable follow-up rate in our sample $(79.3 \%)$, as well as the non-significant differences between women followed and women lost to follow-up,${ }^{15}$ threats to the internal validity of this analysis are minimal.

The results of this study are at risk of bias owing to the large number of women who refused participation. Hence, this bias may have overestimated, or underestimated, the results. In spite of a high refusal rate that threatens the external validity of the results, we consider our sample to be representative of women in Khartoum state because we recruited study subjects from the highest utilised maternity hospital. In addition, parity and educational level estimates from our sample are comparable with state estimates from the latest Sudan census and household survey. ${ }^{17}{ }^{50}$ On the other hand, sociodemographic features of women in Khartoum are different from other states; therefore, the results from this study are not generalisable to all of Sudan.

Acknowledgements The authors are grateful to Ms Rania Adam and the clinical psychologist Ms Fatma Fazaa for their support and help during the follow-up phase in the data collection and clinical interviews.

Contributors DSK was responsible for designing the original study protocol, recruiting and follow-up of study participants, data entry and analysis, and preparation of the initial draft of this article. LL, KG and EB provided constructive criticism on the design of the study, data interpretation and on each draft of this article until the final version was ready for submission.

Funding This research received no specific grant from any funding agency in the public, commercial or not-for-profit sectors.

Competing interests None declared.

\section{Patient consent Obtained.}

Ethics approval Federal Ministry of Health in Sudan and in Norway by Regional Committees for Medical and Health Research Ethics (REK, reference no. 2013/353/REK).

Provenance and peer review Not commissioned; externally peer reviewed.

Data sharing statement No additional data are available.

Open Access This is an Open Access article distributed in accordance with the Creative Commons Attribution Non Commercial (CC BY-NC 4.0) license, which permits others to distribute, remix, adapt, build upon this work noncommercially, and license their derivative works on different terms, provided the original work is properly cited and the use is non-commercial. See: http:// creativecommons.org/licenses/by-nc/4.0/

\section{REFERENCES}

1. WHO. Mental health aspects of women's reproductive health: A global review of the literature. WHO, UNFPA, 2009.

2. EMRO. Maternal, child and adolescent mental health: challenges and strategic directions 2010-2015. WHO, 2010.

3. Weinstock M. The potential influence of maternal stress hormones on development and mental health of the offspring. Brain Behav Immun 2005;19:296-308.

4. Halbreich U, Karkun S. Cross-cultural and social diversity of prevalence of postpartum depression and depressive symptoms. $J$ Affect Disord 2006;91:97-111.

5. Stern G, Kruckman L. Multi-disciplinary perspectives on post-partum depression: an anthropological critique. Soc Sci Med 1983;17:1027-41.

6. Fisher J, Cabral de Mello M, Patel V, et al. Prevalence and determinants of common perinatal mental disorders in women in low- and lower-middle-income countries: a systematic review. Bull World Health Organ 2012;90:139G-49G. 
7. Wachs TD, Black MM, Engle PL. Maternal depression: a global threat to children's health, development, and behavior and to human rights. Child Dev Perspect 2009;3:51-9.

8. Salah TT, Abdelrahman A, Lien L, et al. The mental health of internally displaced persons: an epidemiological study of adults in two settlements in Central Sudan. Int J Soc Psychiatry 2013;59:782-8.

9. Weobong B, Ten Asbroek AH, Soremekun S, et al. Determinants of postnatal depression in rural ghana: findings from the don population based cohort study. Depress Anxiety 2015;32:108-19.

10. Hamdan A, Tamim H. Psychosocial risk and protective factors for postpartum depression in the United Arab Emirates. Arch Womens Ment Health 2011;14:125-33.

11. El-Hachem C, Rohayem J, Bou Khalil RB, et al. Early identification of women at risk of postpartum depression using the Edinburgh Postnatal Depression Scale (EPDS) in a sample of Lebanese women. BMC Psychiatry 2014;14:242.

12. Kakyo TA, Muliira JK, Mbalinda SN, et al. Factors associated with depressive symptoms among postpartum mothers in a rural district in Uganda. Midwifery 2012;28:374-9.

13. Mohammed ES, Mosalem FA, Mahfouz EM, et al. Predictors of postpartum depression among rural women in Minia, Egypt: an epidemiological study. Public Health 2014;128:817-24.

14. Armah B, Yeo D, Kedir BN, et al. MDG 2014 Report: Assessing progress in Africa toward the Millennium Development Goals (Analysis of the Common African Position on the post-2015 Development Agenda). In: United Nations Economic Commission for Africa AU, African Development Bank and United Nations Development Programme. Addis Ababa, Ethiopia: Economic Commission for Africa, 2014, pp 58, 66. http://www.uneca.org/sites/ default/files/PublicationFiles/2014 mdg report.pdf.

15. Khalifa DS, Glavin K, Bjertness E, et al. Postnatal depression among Sudanese women: prevalence and validation of the Edinburgh Postnatal Depression Scale at 3 months postpartum. Int J Womens Health 2015;7:677.

16. Abiodun OA. Postnatal depression in primary care populations in Nigeria. Gen Hosp Psychiatry 2006;28:133-6.

17. Central Bureau of Statistics, Sudan Federal Ministry of Health, Health SFMo. Sudan Household Survey 2010-2nd Round. Khartoum- Sudan. 2010. http://196.29.166.241/CBS-Metadata-env4.2/index.php/catalog/3

18. Ibnouf $\mathrm{AH}$, Borne HW, Maarse JA. Utilization of antenatal care services by Sudanese women in their reproductive age. Saudi Med J 2007;28:737-43.

19. Cox JL, Holden JM, Sagovsky R. Detection of postnatal depression. Development of the 10-item Edinburgh Postnatal Depression Scale. Br J Psychiatry 1987;150:782-6.

20. Cox J, Holden J, Henshaw C. Perinatal mental health. The Edinburg postnatal depression scale (EPDS) manual. 2nd edn. RCPsych Publications, 2014

21. Kaminsky LM, Carlo J, Muench MV, et al. Screening for postpartum depression with the Edinburgh Postnatal Depression Scale in an indigent population: does a directed interview improve detection rates compared with the standard self-completed questionnaire? $J$ Matern Fetal Neonatal Med 2008;21:321-5.

22. Howard LM, Oram S, Galley H, et al. Domestic violence and perinatal mental disorders: a systematic review and meta-analysis. PLoS Med 2013;10:e1001452.

23. Austin MP, Tully L, Parker G. Examining the relationship between lifetime stressful life events and the onset of major depression in Chinese women. J Affect Disord 2007:101:169-74

24. Nongrum $\mathrm{R}$, Thomas $\mathrm{E}$, Lionel $\mathrm{J}$, et al. Domestic violence as a risk factor for maternal depression and neonatal outcomes: a hospital-based cohort study. Indian J Psychol Med 2014; 36:179-81.

25. Alvarez-Segura M, Garcia-Esteve L, Torres A, et al. Are women with a history of abuse more vulnerable to perinatal depressive symptoms? A systematic review. Arch Womens Ment Health 2014:17:343-57.

26. Giri RK, Khatri RB, Mishra SR, et al. Prevalence and factors associated with depressive symptoms among post-partum mothers in Nepal. BMC Res Notes 2015;8:111.
27. Birkeland R, Thompson JK, Phares V. Adolescent motherhood and postpartum depression. J Clin Child Adolesc Psychol 2005;34:292-300

28. Figueiredo B, Dias CC, Brandão S, et al. Breastfeeding and postpartum depression: state of the art review. J Pediatr (Rio J) 2013;89:332-8.

29. Figueiredo B, Canário C, Field T. Breastfeeding is negatively affected by prenatal depression and reduces postpartum depression. Psychol Med 2014;44:927-36.

30. Onsa ZO, Ahmed NMK. Impact of exclusive breast feeding on the growth of Sudanese Children (0-24 Months). Pak J Nutr 2014;13:99-106.

31. Corwin EJ, Murray-Kolb LE, Beard JL. Low hemoglobin level is a risk factor for postpartum depression. $J$ Nutr 2003;133:4139-42.

32. Beard JL, Hendricks MK, Perez EM, et al. Maternal iron deficiency anemia affects postpartum emotions and cognition. J Nutr 2005;135:267-72.

33. Perez EM, Hendricks MK, Beard JL, et al. Mother-infant interactions and infant development are altered by maternal iron deficiency anemia. J Nutr 2005;135:850-5.

34. Albacar G, Sans T, Martín-Santos R, et al. An association between plasma ferritin concentrations measured $48 \mathrm{~h}$ after delivery and postpartum depression. J Affect Disord 2011;131:136-42.

35. Bodnar LM, Wisner KL. Nutrition and depression: implications for improving mental health among childbearing-aged women. Bio Psychiatry 2005;58:679-85.

36. Goshtasebi A, Alizadeh M, Gandevani SB. Association between maternal anaemia and postpartum depression in an urban sample of pregnant women in Iran. $J$ Health Popul Nutr 2013;31:398-402

37. Myint AM, Leonard BE, Steinbusch HW, et al. Th1, Th2, and Th3 cytokine alterations in major depression. $J$ Affect Disord 2005;88:167-73.

38. Hage MP, Azar ST. The link between thyroid function and depression. J Thyroid Res 2012;2012:590648.

39. Abdelrahman EG, Gasim GI, Musa IR, et al. Red blood cell distribution width and iron deficiency anemia among pregnan Sudanese women. Diagn Pathol 2012;7:168.

40. Klainin P, Arthur DG. Postpartum depression in Asian cultures: a literature review. Int J Nurs Stud 2009;46:1355-73.

41. Xie $\mathrm{RH}, \mathrm{He} \mathrm{G}$, Liu A, et al. Fetal gender and postpartum depression in a cohort of Chinese women. Soc Sci Med 2007;65:680-4.

42. Kitamura T, Yoshida K, Okano T, et al. Multicentre prospective study of perinatal depression in Japan: incidence and correlates of antenatal and postnatal depression. Arch Womens Ment Health 2006:9:121-30.

43. Patel V, Rodrigues M, DeSouza N. Gender, poverty, and postnatal depression: a study of mothers in Goa, India. Am J Psychiatry 2002;159:43-7.

44. Ekuklu G, Tokuc B, Eskiocak M, et al. Prevalence of postpartum depression in Edirine, Turkey, and related factors. J Reprod Med 2004;49:908-14.

45. Andajani-Sutjahjo S, Manderson L, Astbury J. Complex emotions, complex problems: understanding the experiances of perinatal depression among new mothers in urban Indonesia. Cult Med Psychiatry 2007:31:101-22

46. Kalyani GHS, Saeed K, Rehman CIU, et al. Incidence of depressive illness in Pakistani women during postnatal period. JColl Physicians Surg Pak 2001:11:246-8.

47. Robertson E, Grace S, Wallington T, et al. Antenatal risk factors for postnatal depression: a prospective study of Chinese women at maternal and child health centres. Gen Hosp Psychiatry 2004;26:289-95.

48. Honikman S, van Heyningen T, Field S, et al. Stepped care for maternal mental health: a case study of the perinatal mental health project in South Africa. PLoS Med 2012;9:e1001222.

49. Hendrick V. Treatment of postnatal depression. BMJ 2003;327:1003-4.

50. Central Bureau of Statistics, Sudan Federal Ministry of Health. Census 2008 results—Khartoum State. CBS, 2013. 